Received: 09.08 .2020

Revised: 22.09 .2020

Accepted: 16.10 .2020

DOI: $10.17804 / 2410-9908.2020 .5 .017-025$

\title{
FORMATION OF A SYSTEM OF INDICATORS FOR EVALUATING THE CHARACTERISTICS OF ASYNCHRONOUS ELECTRIC MOTORS AT THEIR LIFE CYCLE STAGES
}

\author{
S. P. Kurilin ${ }^{1, a)^{*}}$ and A. M. Sokolov ${ }^{2}$ \\ 'Smolensk Branch of the National Research University "Moscow Power Engineering Institute" \\ 1, Energeticheskiy Dr., Smolensk, 2014013, Russian Federation \\ ${ }^{2}$ National Research University "Moscow Power Engineering Institute”, \\ 14, Krasnokazarmennaya St., Moscow, 111250, Russian Federation \\ a) iD https://orcid.org/0000-0001-7843-275X kurilinsp@inbox.ru \\ *Corresponding author. E-mail: kurilinsp@inbox.ru \\ Address for correspondence: Energeticheskiy pr., 1, Smolensk, 2014013, Russian Federation \\ Tel.: +79107128836
}

The article focuses on the development and formation of a system of indicators for assessing the characteristics of asynchronous electric motors (AEMs) at various life cycle stages. The problem is solved on the basis of the topological approach to the analysis of heterogeneous electromechanical systems. The life cycle of AEM and the tasks of its information support are described. The flows of diagnostic information are analyzed. Indicators for assessing the health of AEDs at various stages of their life cycle are proposed.

Keywords: asynchronous electric motor, life cycle, operational states, topological approach, indicators of motor health.

\section{Acknowledgment}

The study was performed under the state assignment (project No. FSWF-2020-0019) and financially supported by the RFBR as part of project No. 20-01-00283.

\section{References}

1. Vol'dek A.I. Elektricheskie mashiny [Electrical Machines]. 3rd ed., Moscow, Al'yans Publ., 2017, 832 p. (In Russian).

2. Gol'dberg O.D., Makarov L.N., Khelemskaya S.P. Inzhenernoe proektirovanie elektricheskikh mashin [Engineering Design of Electrical Machines]. Moscow, Izdat. Dom «Basted» Publ., 2016, 528 p. (In Russian).

3. Kurilin S.P., Denisov V.N. Topologicheskie aspekty teorii asinkhronnykh elektricheskikh mashin [Topological Aspects of the Theory of Asynchronous Electrical Machines]. Smolensk, Universum Publ., 2019, 200 p. (In Russian).

4. Borisov V.V., Kurilin S.P., Chernovalova M.V. Topologicheskii podkhod k issledovaniyu neodnorodnykh elektromekhanicheskikh system. In: Matematicheskie metody $v$ tekhnike i tekhnologiyakh: sb. tr. mezhdunar. nauch. konf. [Mathematical Methods in Engineering and Technology: Proceedings of the International Scientific Conference]. St. Petersburg, Politekhn. Un-t Publ., 2020, vol. 7, pp. 96-99. (In Russian). 
5. Kurilin S.P., Denisov V.N., Fedulov A.S., Dli M.I. Scientific basis of methods for topological diagnostics of asynchronous electric machines. AIP Conference Proceedings, 2018, vol. 2053, pp. 030031. DOI: 10.1063/1.5084392.

6. Kurilin S.P., Denisov V.N. The Development of Topological Diagnostic Methods of Asynchronous Electric Machines. Diagnostics, Resource and Mechanics of materials and structures, 2018, iss. 6, pp. 214-221. DOI: 10.17804/2410-9908.2018.6.214-221. Available at: http://dreamjournal.org/issues/2018-6/2018-6_185.html

7. $\quad$ Kurillin S.P., Denisov V.N., Dli M.I., Bobkov V.I. Vector space as an area of the operation risks characteristics for asynchronous electric machines. IOP Conf. Series: Journal of Physics: Conf. Series, 2019, vol. 1260, pp. 052017. DOI: 10.1088/1742-6596/1260/5/052017.

8. Kurilin S.P., Denisov V.N., Dli M.I., Bobkov V.I. A method for the operational diagnostics of induction motors. AIP Conference Proceedings, 2019, vol. 2176, pp. 040008. DOI: 10.1063/1.5135157. Available at: http://dream-journal.org/issues/2018-6/2018-6_185.html 
Подана в журнал: 09.08.2020

УДК 621.313.333:(512+514.1+515.1)

DOI: $10.17804 / 2410-9908.2020 .5 .017-025$

\title{
ФОРМИРОВАНИЕ СИСТЕМЫ ПОКАЗАТЕЛЕЙ ДЛЯ ОЦЕНКИ ХАРАКТЕРИСТИК АСИНХРОННЫХ ЭЛЕКТРОДВИГАТЕЛЕЙ НА ЭТАПАХ ЖИЗНЕННОГО ЦИКЛА
}

\author{
С. П. Курилин ${ }^{1)^{*}}$, А. М. Соколов ${ }^{2)}$ \\ ${ }^{1}$ Филиал ФГБОУ ВО «НИУ «МЭИ» в г. Смоленске, \\ 1, Энергетический пр., Смоленск, 214013, Российская Федерация \\ ${ }^{2}$ ФГБОУ ВО «НИУ «МЭИ», \\ 14, ул. Красноказарменная, Москва, 111250, Российская Федераџия \\ a) iD https://orcid.org/0000-0001-7843-275X kurilinsp@inbox.ru \\ *Ответственный автор. Электронная почта: kurilinsp@inbox.ru
}

Адрес для переписки: д. 1, Энергетический пр., г. Смоленск, 214013, Российская Федерация, Тел.: +7-910-712-88-36

Статья посвящена выработке и формированию системы показателей для оценки характеристик асинхронных электродвигателей (АЭД) на различных этапах жизненного цикла. Задача решается на основе топологического подхода к анализу неоднородных электромеханических систем. Описывается жизненный цикл АЭД и задачи его информационного сопровождения. Представлены потоки диагностической информации. Предложены показатели для оценки технического состояния АЭД на различных этапах жизненного цикла.

Ключевые слова: асинхронный электродвигатель, жизненный цикл, эксплуатационные состояния, топологический подход, показатели технического состояния.

\section{1. Введение}

Асинхронные электродвигатели (АЭД) характеризуются массовым применением и входят в состав большинства технических систем. О широте их применения свидетельствует тот факт, что эксплуатируемый в настоящее время парк АЭД Российской Федерации имеет мощность порядка 70 млн кВт.

Применяемые в настоящее время показатели технического и энергетического качества АЭД, такие как КПД и коэффициент мощности $[1,2]$, предполагают параметрическую однородность объекта. К тому же они являются зависимыми, т. е. характеризуют как техническое состояние самого объекта, так и режим его эксплуатации. Вместе с тем по своим физическим и параметрическим характеристикам АЭД является неоднородной электромеханической системой.

Отметим, что у параметрически неоднородного электродвигателя могут быть и однородные эксплуатационные состояния. При этом сам асинхронный электродвигатель как физико-техническая система параметрически неоднороден. Подтверждением этого, в частности, является факт различных входных сопротивлений электродвигателя для токов прямой и нулевой последовательностей. Параметрическая неоднородность, в основе которой лежит физическая неоднородность устройства, может проявляться или не проявляться, но она внутренне присуща электромеханической системе и неотъемлема от нее.

Значение факторов качественного проектирования и регулярной эксплуатационной диагностики в повышении технического уровня и надежности технических систем весьма высоко. Это связано с укрупнением технических систем, высокой степенью их интегрированности и с повышением их роли в экономической жизни страны. В полной мере это отно- 
сится к качественному проектированию и эксплуатационной диагностике АЭД, которые в значительной мере определяют технический уровень, надежность и живучесть технических систем. При этом ключевую роль играют, во-первых, система показателей для оценки характеристик АЭД на различных этапах жизненного цикла, во-вторых, методы и способы их мониторинга, производимого по ходу эксплуатации. В этих аспектах факт параметрической неоднородности электродвигателя, проявляющийся по мере его эксплуатационного старения, является существенным.

\section{2. Постановка задачи и методы решения}

Задачей исследования является выработка и формирование системы показателей для оценки характеристик асинхронных электродвигателей на различных этапах жизненного цикла. Разрабатываемые показатели должны быть независимыми от внешних воздействий, т. е. должны отображать само изделие, а не режим его эксплуатации.

Поставленная задача решается методами, способами и средствами, присущими топологическому подходу к анализу неоднородных электромеханических систем [3-5]. В соответствии с ним разрабатываемые показатели отображают состояние векторного пространства АЭД и те изменения, которые в нем происходят в связи с эксплуатационным старением изделия.

\section{3. Результаты и обсуждение}

\section{1. Жизненный цикл АЭД}

В жизненном цикле АЭД выделяются фазы проектирования, изготовления и эксплуатации изделия. На протяжении фазы эксплуатации состояния АЭД изменяются от эталонного состояния, имеющего место в момент выпуска с производящего предприятия, до предельного состояния, имеющего место в момент прекращения эксплуатации. Фазы жизненного цикла и эксплуатационные состояния АЭД на оси времени жизненного цикла представлены на рис. 1.

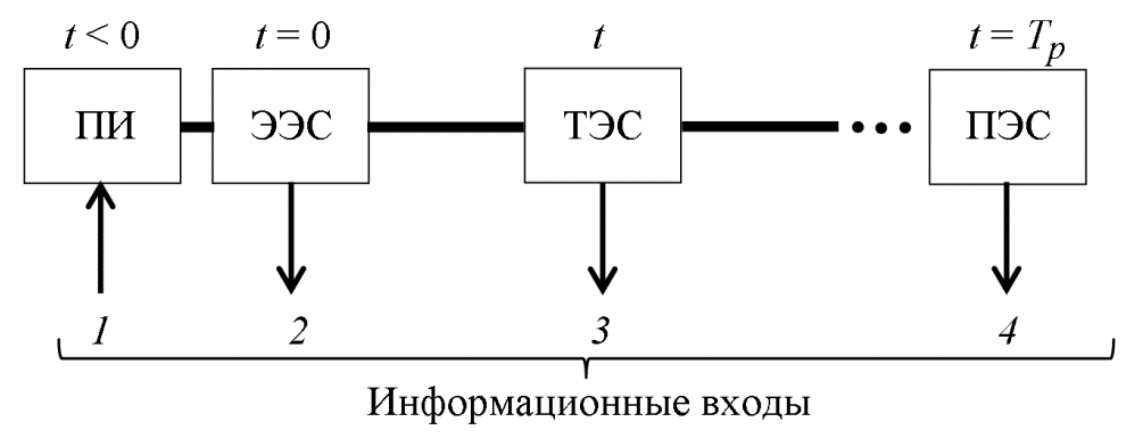

Рис. 1. Фазы жизненного цикла и эксплуатационные состояния АЭД

Здесь ПИ - фаза проектирования и изготовления изделия, имеющая место до начала эксплуатации АЭД. По информационному входу 1 поступают обработанные данные диагностических тестирований и учетные данные эксплуатируемых АЭД. ЭЭС - эталонное эксплуатационное состояние, в котором все технические показатели АЭД соответствуют требованиям нормативно-технической документации, а само изделие полностью работоспособно. ЭЭС соответствует моменту $t=0$ завершения выходных испытаний и начала эксплуатации АЭД. По входу 2 в процессе выходных испытаний снимается информация об эталонном техническом состоянии изделия. Вероятность работоспособности для ЭЭС принимается равной единице. ТЭС - текущее эксплуатационное состояние, соответствующее фиксированному моменту эксплуатации $t>0$. 
В этом состоянии АЭД уже подвергся эксплуатационному старению и, возможно, приобрел мелкие (не вызывающие потерю работоспособности) повреждения. В связи с этим технические показатели изделия отклонились от эталонных значений ЭЭС, что можно трактовать как частичную утрату работоспособности. По входу 3 в процессе эксплуатационного тестирования снимается информация о текущем техническом состоянии изделия. ПЭС - предельное эксплуатационное состояние, соответствующее моменту прекращения эксплуатации $t=T_{p}$. В предельном состоянии технические показатели АЭД неустранимо ушли за рамки требований нормативнотехнической документации. В нормативно-техническом, смысле изделием полностью утрачена работоспособность и вероятность сохранения работоспособности равна нулю. По показателям ПЭС определяется и передается по входу 4 информация о предельных отклонениях технических показателей от эталонных значений.

\section{2. Задачи информационного сопровождения жизненного цикла АЭД}

В плане научного и информационного сопровождения жизненного цикла АЭД выделяются две задачи - прогнозная и структурно-аналитическая.

Содержанием прогнозной задачи является получение сведений о текущей работоспособности АЭД, в частности - получение прогнозных оценок остаточного ресурса и вероятности потери работоспособности. Оценки даются для каждого электродвигателя и впоследствии анализируются и обобщаются по характерным эксплуатационным группам. Источником информации для решения прогнозной задачи служат данные о текущем техническом состоянии эксплуатируемого АЭД, получаемые в результате регулярного диагностического тестирования. Выходом задачи является принятие решения о продолжении или прекращении его эксплуатации. Возможно также решение об изменении режима эксплуатации.

Содержанием структурно-аналитической задачи является, во-первых, разделение эксплуатируемых АЭД по характерным эксплуатационным группам, во-вторых, получение сведений о степени соответствия конструктивных и технологических решений, принятых на этапе проектирования и изготовления изделия, условиям и режимам эксплуатации. Оценки соответствия даются для каждой фиксированной эксплуатационной группы.

Источниками информации для решения структурно-аналитической задачи служат обобщенные данные диагностических тестирований, а также результаты моделирования эксплуатационных состояний АЭД. Выходом задачи являются проектно-конструкторские и технологические рекомендации, а также эксплуатационные требования для фиксированной эксплуатационной группы АЭД.

Таким образом, эксплуатационная диагностика связывает воедино проектировочные решения и производственные технологии - с одной стороны и вероятность безотказной работы, и остаточный ресурс - с другой стороны. Естественным продолжением такого объединения является выработка критериев оптимальности жизненного цикла электромеханических систем, а также создание методов, способов, правил и технологий их реализации в рамках фиксированной эксплуатационной группы.

\section{3. Эксплуатационные группы АЭД}

Основной физический процесс, отслеживаемый в ходе эксплуатационной диагностики АЭД, процесс его эксплуатационного старения, т. е. процесс постепенной утраты изделием физических, конструктивных, параметрических и технических свойств. Эксплуатационное старение - сложный и трудно формализуемый процесс, зависящий от многих детерминированных и случайных факторов. Вместе с тем, очевидно, что интенсивность старения определяется степенью защиты, а также условиями и характером эксплуатации изделия, т. е. длительностью эксплуатации, влажностью, запыленностью, агрессивностью окружающей среды, частотой и интенсивностью перегрузок, сезонностью эксплуатации, нестабильностью пара- 
метров питания и др. В этой связи авторы считают целесообразным введение специального термина «эксплуатационная группа», под которой понимается группа АЭД с приблизительно одинаковыми условиями и характером эксплуатации и, следовательно, с приблизительно одинаковыми темпами эксплуатационного старения. Показатель темпа эксплуатационного старения, определяемый в ходе эксплуатационной диагностики, может быть использован как числовой критерий для отнесения АЭД к той или иной эксплуатационной группе.

\section{4. Информационные потоки на этапах жизненного цикла АЭД}

Информация, передаваемая по входам 1-4 (рис. 1), обрабатывается с целью получения необходимых прогнозных и структурно-аналитических показателей АЭД. Диаграмма информационных потоков на этапах жизненного цикла АЭД представлена на рис. 2.

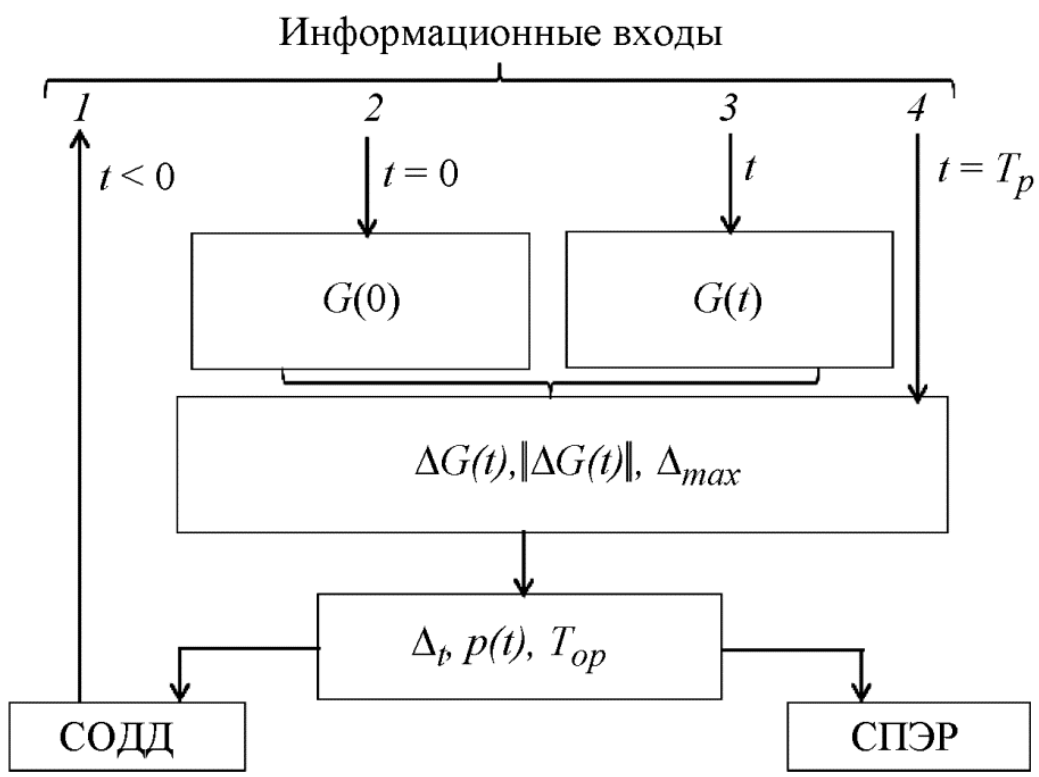

Рис. 2. Информационные потоки на этапах жизненного цикла АЭД

Методология проведения диагностического обследования и способ формирования диагностических матриц приведены в [4, 6-8]. В соответствии с ними на первом этапе диагностического обследования экспериментально формируются матрицы Грина для состояний ЭЭС и ТЭС. Матрица Грина для состояния ЭЭС в долях от амплитуды тока проводимости имеет вид:

$$
G(0)=\left(\begin{array}{lll}
1 & 0 & 1 \\
1 & 1 & 0 \\
0 & 1 & 1
\end{array}\right)
$$

Матрица Грина для состояния ТЭС в тех же единицах имеет вид

$$
G(t)=\left(\begin{array}{lll}
I_{A m} & i_{A m} & I_{C m} \\
I_{A m} & I_{B m} & i_{B m} \\
i_{C m} & I_{B m} & I_{C m}
\end{array}\right),
$$


где $I_{A m} ; I_{B m} ; I_{C m}$ - амплитуды токов проводимости в фазах обмотки статора A, B, C; $i_{A m} ; i_{B m}$; $i_{C m}$ - амплитуды индуцированных токов в фазах обмотки статора А, B, С. Матрицы $G(0)$ и $G(t)$ формируются в процессах выходного и эксплуатационного тестирования АЭД соответственно.

\section{5. Показатели технического состояния АЭД}

На втором этапе диагностического обследования вычисляется матрица текущих отклонений (рис. 2):

$$
\Delta G(t)=\operatorname{abs}(G(0)-G(t))
$$

которая далее нормируется

$$
\|\Delta G(t)\|=a b s\left(\begin{array}{ccc}
\frac{1-I_{A m}}{\Delta I} & \frac{i_{A m}}{\Delta i} & \frac{1-I_{C m}}{\Delta I} \\
\frac{1-I_{A m}}{\Delta I} & \frac{1-I_{B m}}{\Delta I} & \frac{i_{B m}}{\Delta i} \\
\frac{i_{C m}}{\Delta i} & \frac{1-I_{B m}}{\Delta I} & \frac{1-I_{C m}}{\Delta I}
\end{array}\right) .
$$

Для нормирования вводятся различные предельные отклонения элементов проводимости $(\Delta I)$ и индуктивных элементов $(\Delta i)$ матрицы текущих отклонений. Предельные отклонения определяются экспертным путем и ассоциируются с предельным эксплуатационным состоянием. Это означает, что достижение каким-либо элементом матрицы $\|\Delta G(t)\|$ значения 1 свидетельствует о появлении состояния ПЭС и влечет за собой прекращение эксплуатации АЭД.

После нормирования определяется максимальное нормированное отклонение элементов матрицы Грина от эталонных значений свидетельствующее о степени эксплуатационного старения изделия:

$$
\Delta_{\max }=\max \|\Delta G(t)\| .
$$

На третьем этапе диагностического обследования определяются:

- показатель темпа эксплуатационного старения изделия

$$
\Delta_{t}=\frac{\Delta_{\max }}{t} ;
$$

- показатель вероятности сохранения работоспособности изделия

$$
p(t)=1-\Delta_{\text {max }} ;
$$

- показатель остаточного ресурса изделия

$$
T_{o p}=\frac{p(t)}{\Delta_{t}} .
$$


Данные показатели направляются в систему поддержки эксплуатационных решений (СПЭР), а также в систему обработки диагностических данных (СОДД). СПЭР накапливает и обрабатывает информацию для принятия решения о продолжении или прекращении эксплуатации изделия. СОДД обрабатывает диагностические и учетные данные обследованного АЭД и относит его к определенной эксплуатационной группе.

Полученная в ходе периодических диагностических обследований информация позволяет обоснованно решать эксплуатационные задачи и в отношении конкретного АЭД, и в отношении эксплуатационных групп, и в отношении парков АЭД различных технических систем и отраслей экономики. Отметим также, что в соответствии со спецификой формирования матриц Грина, информация, содержащаяся в них, не соотносится с режимом эксплуатации АЭД, т. е. является безусловной и характеризует только само изделие.

\section{4. Заключение}

В плане научного и информационного сопровождения жизненного цикла АЭД выделяются две задачи - прогнозная и структурно-аналитическая. Их решение позволяет организовать эффективную эксплуатацию парка АЭД РФ. При этом ключевую роль играют, вопервых, система показателей для оценки характеристик АЭД на различных этапах жизненного цикла, во-вторых, методы и способы их мониторинга, производимого по ходу эксплуатации. В этих аспектах существенным является факт параметрической неоднородности электродвигателя, проявляющийся по мере его эксплуатационного старения.

Эксплуатационная диагностика является общим звеном отмеченных выше задач. На основе данных диагностического тестирования определяются такие показатели текущего технического состояния АЭД, как показатель темпа эксплуатационного старения, показатель вероятности сохранения работоспособности и показатель остаточного ресурса. Данные показатели позволяют существенно повысить обоснованность принимаемых эксплуатационных решений.

\section{Благодарность}

Исследование выполнено при финансовой поддержке РФФИ в рамках научного проекта № 20-01-00283, и в рамках государственного задания, проект № FSWF-2020-0019.

\section{Литература}

1. Вольдек А. И. Электрические машины. - 3-е изд. - М. : Альянс, 2017. - 832 с.

2. Гольдберг О. Д., Макаров Л. Н., Хелемская С. П. Инженерное проектирование электрических машин. - М. : Издат. дом «Бастед», 2016. - 528 с.

3. Курилин С. П., Денисов В. Н. Топологические аспекты теории асинхронных электрических машин. - Смоленск : Универсум, 2019. - 200 с.

4. Борисов В. В., Курилин С. П., Черновалова М. В. Топологический подход к исследованию неоднородных электромеханических систем // Математические методы в технике и технологиях : сб. тр. междунар. науч. конф. - СПб. : Изд-во Политехн. ун-та, 2020. - Т. 7. C. 96-99.

5. Scientific basis of methods for topological diagnostics of asynchronous electric machines / S. P. Kurilin, V. N. Denisov, A. S. Fedulov, M. I. Dli // AIP Conference Proceedings. - 2018. Vol. 2053. - P. 030031. - DOI: 10.1063/1.5084392.

6. Kurilin S. P., Denisov V. N. The Development of Topological Diagnostic Methods of Asynchronous Electric Machines // Diagnostics, Resource and Mechanics of materials and structures. 2018. - Iss. 6. - P. 214-221. - DOI: 10.17804/2410-9908.2018.6.214-221. - URL: http://dreamjournal.org/issues/2018-6/2018-6_185.html 
7. Vector space as an area of the operation risks characteristics for asynchronous electric machines / S. P. Kurillin, V. N. Denisov, M. I. Dli, V. I. Bobkov // IOP Conf. Series. Journal of Physics. Conf. Series. - 2019. - Vol. 1260. - P. 052017. - DOI:10.1088/1742-6596/1260/5/052017.

8. A method for the operational diagnostics of induction motors / S. P. Kurilin, V. N. Denisov, M. I. Dli, V. I. Bobkov // AIP Conference Proceedings. - 2019. - Vol. 2176. - P. 040008. DOI: 10.1063/1.5135157. - URL: http://dream-journal.org/issues/2018-6/2018-6_185.html 